\title{
SPECTRAL SYNTHESIS AND APPLICATIONS TO $C_{0}$-GROUPS
}

\author{
M. ZARRABI
}

(Received 22 February 1993; revised 6 May 1993)

Communicated by I. Raeburn

\section{Abstract}

Let $k \geq 0$ be an integer, $T=(T(t))_{t \in \mathbb{R}}$ a $C_{0}$-group of bounded operators and $A$ the infinitesimal generator of $T$. We prove that if,

$$
\|T(t)\|=o\left(t^{k+1}\right) \quad \text { and } \quad \log ^{+}\|T(-t)\|=o\left(t^{1 / 2}\right) \quad(t \rightarrow+\infty),
$$

and if the spectrum of $A$ is equal to $\{\lambda\}$, then $A$ is bounded and $(A-\lambda)^{k+1}=0$. Examples are given to show that these conditions are, essentially, the best possible.

1991 Mathematics subject classification (Amer. Math. Soc.): 47D03.

\section{Introduction}

Let $X$ be a Banach space and let $T: \mathbb{R} \rightarrow L(X)$ be a $C_{0}$-group, that is, $T$ satisfies the following conditions:

(i) $T(0)=I$, where $I$ is the identity operator;

(ii) $T(t+s)=T(t) T(s) \quad(t, s \in \mathbb{R})$;

(iii) For each $x \in \mathbb{R}$, the map $t \rightarrow T(t) x$ is norm-continuous from $\mathbb{R}$ into $X$.

Let $\mathscr{D}$ be the set of all $x \in X$ such that the limit

$$
A x=\lim _{t \rightarrow 0} \frac{T(t) x-x}{t}
$$

exists. Then $\mathscr{D}$ is a linear space dense in $X$ and $A$ is a closed linear operator with domain $\mathscr{D}[11$, p. 5$] ; A$ is called the infinitesimal generator of $T$.

In this paper we consider the following problem: Under what conditions on $T$ do we have $(A-\lambda)^{k+1}=0$, when the spectrum of $A, \sigma(A)$, is equal to $\{\lambda\}$ and where $k \geq 0$ is a given integer?

(c) 1996 Australian Mathematical Society 0263-6115/95 \$A2.00+0.00 
We prove that if $T$ satisfies the conditions

(a)

$$
\begin{aligned}
& \|T(t)\|=o\left(t^{k+1}\right) \quad(t \rightarrow+\infty), \\
& \log ^{+}\|T(-t)\|=o\left(t^{1 / 2}\right) \quad(t \rightarrow+\infty),
\end{aligned}
$$

and if $\sigma(A)=\{\lambda\}$, then $A$ is bounded and $(A-\lambda)^{k+1}=0$.

We obtain this result by using spectral sythesis arguments in weighted algebras. For $k=0$ we give the equivalence between the above result and the spectral synthesis property for points. We prove that if $\omega$ is a weight on $\mathbb{R}$ then points satisfy the $\omega$-spectral synthesis if and only if for all $C_{0}$-groups $T$ dominated by $\omega$ such that $\sigma(A)=\{\lambda\}$, we have $A=\lambda I$.

For the analogous problem in the discrete case, some results are known. Let $R$ be a bounded and invertible operator on a Banach space with spectrum equal to $\{\lambda\}$. Gelfand proved that if the sequence $\left(R^{n}\right)_{n \in \mathbb{Z}}$ is bounded then $R=\lambda I$ (see [1, 12]). This result is extended in [2] and [14] to contractions $R$ such that

$$
\log ^{+}\left\|R^{-n}\right\|=o\left(n^{\frac{1}{2}}\right) \quad(n \rightarrow+\infty) .
$$

More generally, it is proved in [2] that if $\left(b^{\prime}\right)$ and

(c) $\left\|R^{n}\right\|=O\left(n^{k}\right) \quad(n \rightarrow+\infty)$

hold, then $(R-\lambda)^{k+1}=0$. We improve this result by replacing (c) by the condition (a') $\quad\left\|R^{n}\right\|=o\left(n^{k+1}\right) \quad(n \rightarrow+\infty)$.

Finally, we construct examples to show that (a), (b) and $\left(a^{\prime}\right),\left(b^{\prime}\right)$ are, essentially, the best growth conditions for the two results.

\section{Entire functions of exponential type zero}

Let $f$ be an entire function. Then $f$ is said to be of exponential type if there exists a constant $c>0$ such that $|f(z)|=O\left(e^{c|z|}\right)(z \in \mathbb{C})$.

The infimum $\tau$ of all constants $c$ for which the above inequality holds is called the exponential type of $f$. It is easy to see that

$$
\tau=\limsup _{|z| \rightarrow+\infty} \frac{\log |f(z)|}{|z|} .
$$

THEOREM 2.1. Let $f$ be an entire function of exponential type zero. If $f$ is bounded on $[0,+\infty)$ and satisfies $\log ^{+}|f(-t)|=o\left(t^{1 / 2}\right)(t \rightarrow+\infty)$, then $f$ is constant.

PROOF. Set, for $z \in \mathbb{C} \backslash(-\infty, 0], \log z=\log |z|+i \operatorname{Arg}(z)$, where $\operatorname{Arg}(z)$ is the determination of the argument of $z$ which belongs to $(-\pi,+\pi]$. The function $z \rightarrow z^{1 / 2}=e^{(\log z) / 2}$ is analytic on $\mathbb{C} \backslash(-\infty, 0]$ and continuous on $\mathbb{C} \backslash(-\infty, 0)$. Denote by $\mathbb{C}_{+}$the right-hand half plane $\{z \in \mathbb{C}: \operatorname{Re} z>0\}$. Let $\epsilon>0$ and set

$$
g_{\epsilon}(z)=e^{-\epsilon z^{1 / 2}} f(i z) \quad\left(z \in \overline{\mathbb{C}}_{+}\right) .
$$


Then $g_{\epsilon}$ is analytic on $\mathbb{C}_{+}$and continuous on $\overline{\mathbb{C}}_{+}$. We have

$$
\left|g_{\epsilon}(z)\right|=e^{-\epsilon \operatorname{Re}\left(z^{1 / 2}\right)}|f(i z)|=e^{-\epsilon|z|^{1 / 2} \cos \left(\frac{1}{2} \operatorname{Arg} z\right)}|f(i z)| .
$$

For $z \in \mathbb{C}_{+}, \cos \left(\frac{1}{2} \operatorname{Arg} z\right) \geq 0$ and so $\left|g_{\epsilon}(z)\right| \leq|f(i z)|$. Since $f$ is of exponential type zero, we have for an arbitrary $a>0$,

$$
\left|g_{\epsilon}(z)\right|=O\left(e^{a|z|}\right) \quad(|z| \rightarrow+\infty, \operatorname{Re} z \geq 0) .
$$

Moreover, we obtain for $t \in \mathbb{R},\left|g_{\epsilon}(i t)\right|=e^{-\epsilon(|t| / 2)^{1 / 2}}|f(-t)|$. The hypothesis on $f$ implies that there exists a constant $m_{\epsilon}>0$ such that $|f(t)| \leq m_{\epsilon} e^{\epsilon(t \mid / 2)^{1 / 2}}(t \in \mathbb{R})$. Thus $g_{\epsilon}$ is bounded by $m_{\epsilon}$ on the imaginary axis. It follows from [4, Theorem 1.4.3] that $g_{\epsilon}$ is bounded by $m_{\epsilon}$ on $\overline{\mathbb{C}}_{+}$. We have

$$
|f(i z)|=\left|e^{\epsilon z^{1 / 2}} g_{\epsilon}(z)\right|=e^{\epsilon|z|^{1 / 2} \cos \left(\frac{1}{2} \operatorname{Arg} z\right)}\left|g_{\epsilon}(z)\right| \leq m_{\epsilon} e^{\epsilon|z|^{1 / 2}} .
$$

So

$$
\limsup _{|z| \rightarrow+\infty, \operatorname{Re} z \geq 0} \frac{\log |f(i z)|}{|i z|^{1 / 2}}=0 .
$$

Applying the same method to the function $f(-z)$, we obtain

$$
\limsup _{|z| \rightarrow+\infty, \operatorname{Re} z \leq 0} \frac{\log |f(i z)|}{|i z|^{1 / 2}}=0 .
$$

Finally, the function $f$ is of growth $(1 / 2,0)$ in the sense of $[4$, Chapter 2] and bounded on $[0,+\infty)$. Hence $f$ is constant by [4, Theorem 3.1.5].

COROLlaRY 2.2. Let $k \geq 0$ be an integer and let $f$ be an entire function of exponential type zero. If $f$ satisfies the two following conditions,

(i) $f(t)=o\left(t^{k+1}\right) \quad(t \rightarrow+\infty)$,

(ii) $\log ^{+}|f(t)|=o\left(t^{\frac{1}{2}}\right) \quad(t \rightarrow+\infty)$,

then $f$ is a polynomial of degree $\leq k$.

PROOF. Consider the function

$$
g(z)=\frac{1}{z^{k+1}}\left(f(z)-\sum_{i=0}^{k} \frac{f^{(i)}(0)}{i !} z^{i}\right) .
$$

It is easily verified that $g$ satisfies the hypothesis of Theorem 1.1 and thus $g$ is constant. It follows from the definition of $g$ that $f$ is a polynomial of degree $\leq k+1$, and from condition (i) that $f$ is polynomial of degree $\leq k$. 


\section{Spectral synthesis in weighted algebras}

Let $\omega$ be a continuous function on $\mathbb{R}$ such that $\omega(t) \geq 1$ and $\omega(t+s) \leq$ $\omega(t) \omega(s)(t, s \in \mathbb{R})$. The function $\omega$ is called a weight.

Denote by $M_{\omega}(\mathbb{R})$ the space of all complex-valued measures $\mu$ on $\mathbb{R}$ such that

$$
\|\mu\|_{\omega}=\int_{-\infty}^{+\infty} \omega(t) d|\mu|(t)<+\infty
$$

where $|\mu|$ is the total variation of $\mu$. Let $\mu, v \in M_{\omega}(\mathbb{R})$. By the Riesz representation theorem there exists a unique measure, denoted $\mu \star \nu$ and called the convolution product of $\mu$ and $\nu$, such that

$$
\int_{-\infty}^{+\infty} \int_{-\infty}^{+\infty} f(s+t) d \mu(s) d \nu(t)=\int_{-\infty}^{+\infty} f(s) d(\mu \star \nu)(s)
$$

for all continuous functions $f$ on $\mathbb{R}$ which vanish at infinity. We have $\mu \star \nu \in M_{\omega}(\mathbb{R})$ and $\|\mu \star v\|_{\omega} \leq\|\mu\|_{\omega}\|\nu\|_{\omega}$.

The space $M_{\omega}(\mathbb{R})$ with convolution product and norm $\|\cdot\|_{\omega}$ is a unital Banach algebra (see [9, Sec. 4.16]). If we denote by $\delta_{t}$ the Dirac measure concentrated at $\{t\}$, then $\delta_{0}$ is the unit of $M_{\omega}(\mathbb{R})$ and $\left\|\delta_{t}\right\|_{\omega}=\omega(t)(t \in \mathbb{R})$.

Set $L_{\omega}(\mathbb{R})$ to be the space of all measurable functions $f$ such that

$$
\|f\|_{\omega}=\int_{-\infty}^{+\infty}|f(t)| \omega(t) d t<+\infty
$$

The space $L_{\omega}(\mathbb{R})$ is naturally identifiable with a closed ideal of $M_{\omega}(\mathbb{R})$. The convolution product of $f, g \in L_{\omega}(\mathbb{R})$ is defined almost everywhere in the formula

$$
(f \star g)(s)=\int_{-\infty}^{+\infty} f(t) g(s-t) d t .
$$

The dual of $L_{\omega}(\mathbb{R})$, denoted by $L_{\omega^{-1}}^{\infty}(\mathbb{R})$, is the set of all measurable functions $g$ such that $\operatorname{esssup}_{t \in \mathbb{R}}|g(t)| / \omega(-t)<+\infty$, and the duality is implemented by the formula

$$
\langle f, g\rangle=\int_{-\infty}^{+\infty} f(t) g(-t) d t \quad\left(f \in L_{\omega}(\mathbb{R}), g \in L_{\omega^{-1}}^{\infty}(\mathbb{R})\right) .
$$

We say the weight $\omega$ is regular if

$$
\int_{-\infty}^{+\infty} \frac{\log \omega(t)}{1+t^{2}} d t<+\infty
$$


It is well-known that the algebra $L_{\omega}(\mathbb{R})$ is regular in the sense of $[10, \mathrm{pp} .221,226]$ if and only if the weight $\omega$ is regular [10, p. 118].

For $\mu \in M_{\omega}(\mathbb{R})$ we denote by $\hat{\mu}$ the Fourier transformation of $\mu$, so that

$$
\hat{\mu}(x)=\int_{-\infty}^{+\infty} e^{-i x t} d \mu(t) \quad(x \in \mathbb{R}) .
$$

If $I$ is a closed ideal of $L_{\omega}(\mathbb{R})$, the hull of $I$ is the set $h(I)=\{x \in \mathbb{R}: \hat{f}(x)=0(f \in$ I)\}.

Let $E$ be a closed subset of $\mathbb{R}$. We set $I_{\omega}(E)=\left\{f \in L_{\omega}(\mathbb{R}): \hat{f}_{\mid E}=0\right\}$, and we denote by $J_{\omega}(E)$ the closure, with respect to the norm $\|.\|_{\omega}$, of the set $\left\{f \in L_{\omega}(\mathbb{R})\right.$ : $\hat{f}=0$ on some neighbourhood of $E\}$. It is easily seen that $I_{\omega}(E)$ and $J_{\omega}(E)$ are closed ideals of $L_{\omega}(\mathbb{R})$. If the weight $\omega$ is regular then $h\left(I_{\omega}(E)\right)=h\left(J_{\omega}(E)\right)=E$ and $J_{\omega}(E) \subseteq I \subseteq I_{\omega}(E)$ for all closed ideals $I$ of $L_{\omega}(\mathbb{R})$ such that $h(I)=E[10, \mathrm{p}$. 224].

DEFINITION 3.1. Let $\omega$ be a regular weight and let $E$ be a closed subset of $\mathbb{R}$. Then

(i) E satisfies $\omega$-synthesis if $J_{\omega}(E)=I_{\omega}(E)$;

(ii) $f$ satisfies $\omega$-synthesis for $E$ if $f \in J_{\omega}(E)$.

Thus $E$ satisfies the $\omega$-synthesis when there exists exactly one closed ideal in $L_{\omega}(\mathbb{R})$ with hull $E$.

In [13] it is proved that closed countable subsets of $\mathbb{R}$ satisfy the $\omega$-synthesis for all weights $\omega$ such that

$$
\begin{cases}\omega(t)=1 & (t \geq 0), \\ \log \omega(-t)=o\left(t^{\frac{1}{2}}\right) & (t \rightarrow+\infty) .\end{cases}
$$

THEOREM 3.2. Let $x \in \mathbb{R}$, let $k \geq 0$ be an integer and let $\omega$ be a weight such that:

(i) $\omega(t)=o\left(t^{k+1}\right) \quad(t \rightarrow+\infty)$;

(ii) $\log \omega(-t)=o\left(t^{\frac{1}{2}}\right) \quad(t \rightarrow+\infty)$;

(iii) $\liminf _{|t| \rightarrow+\infty} \omega(t) /\left(1+|t|^{k}\right)>0$.

Then a function $f \in L_{\omega}(\mathbb{R})$ satisfies $\omega$-synthesis for $\{x\}$ if and only if $\hat{f}^{(i)}(x)=0$ for $i=0,1, \ldots, k$.

PROOF. By using the transformation $f(t) \rightarrow e^{i x t} f(t)$ we can suppose that $x=0$. Assume that $f$ satisfies the $\omega$-synthesis for $\{0\}$. Let $\left(f_{n}\right)_{n \geq 0}$ be a sequence in $L_{\omega}(\mathbb{R})$ such that for each integer $n, \hat{f}_{n}$ vanishes on a neighborhood of $\{0\}$ and $\left\|f_{n}-f\right\|_{\omega} \rightarrow 0$ as $n \rightarrow+\infty$. Condition (iii) ensures the existence of the first $k$ derivatives for all 
Fourier transforms of elements of $L_{\omega}(\mathbb{R})$, and we have for $i=0, \ldots, k \hat{f}_{n}^{(i)}(0)=0$, $\hat{f}_{n}^{(i)}(0) \rightarrow \hat{f}^{(i)}(0)$ as $n \rightarrow+\infty$, and so $\hat{f}^{(i)}(0)=0$.

Conversely, let $\pi$ be the canonical surjection from $L_{\omega}(\mathbb{R})$ onto $L_{\omega}(\mathbb{R}) / J_{\omega}(\{0\})$. There exists $p \in L_{\omega}(\mathbb{R})$ such that $\pi(p)$ is the unit of the quotient algebra $L_{\omega}(\mathbb{R}) / J_{\omega}\{(0)\}$ (see [13, Proposition 1.2]).

For all $t \in \mathbb{R}$ set $\pi\left(\delta_{t}\right)=\pi\left(p \star \delta_{t}\right) ;\left(\pi\left(\delta_{t}\right)\right)_{t \in \mathbb{R}}$ is a norm-continuous $C_{0}$-group, and so there exists $u=\lim _{t \rightarrow+\infty}\left(\pi\left(\delta_{t}\right)-\pi\left(\delta_{0}\right)\right) / t$ such that $\pi\left(\delta_{t}\right)=e^{t u}(t \in \mathbb{R})[9$, Theorem 9.4.2].

The weight $\omega$ is regular and so $h\left(J_{\omega}(\{0\})\right)=\{0\}$. Thus the set of characters of the algebra $L_{\omega}(\mathbb{R}) / J_{\omega}(\{0\})$ is equal to $\left\{\chi_{0}\right\}$, where $\chi_{0}(\pi(f))=\hat{f}(0),\left(f \in L_{\omega}(\mathbb{R})\right)$. Hence the spectrum of $u$ equals $\left\{\chi_{0}(u)\right\}=\{0\}$ and so $\lim _{n \rightarrow+\infty}\left\|u^{n}\right\|^{1 / n}=0$. Hence, for each $\epsilon>0$, there exists a constant $m_{\epsilon}>0$ such that $\left\|u^{n}\right\| \leq m_{\epsilon} \epsilon^{n}(n \geq 0)$.

Set $\varphi(z)=e^{z u}(z \in \mathbb{C})$. Then,

$$
\|\varphi(z)\| \leq \sum_{n=0}^{+\infty} \frac{\left\|u^{n}\right\||z|^{n}}{n !} \leq m_{\epsilon} \sum_{n=0}^{+\infty} \frac{\epsilon^{n}|z|^{n}}{n !}=m_{\epsilon} e^{\epsilon|z|}
$$

and so $\varphi(z)$ is a vector-valued entire function of exponential type zero. Moreover $\varphi(t)=\pi\left(\delta_{t}\right)(t \in \mathbb{R})$, and so

$$
\|\varphi(t)\|=\left\|\pi\left(\delta_{t}\right)\right\|=\left\|\pi\left(p \star \delta_{t}\right)\right\| \leq\|p\|_{\omega} \omega(t) .
$$

It follows from the Hahn-Banach theorem and from Corollary 2.2 that $\varphi(z)$ is a polynomial of degree $\leq k$. Let $a_{0}, \ldots, a_{k} \in L_{\omega}(\mathbb{R}) / J_{\omega}(\{0\})$ be such that

$$
\varphi(z)=a_{0}+a_{1} z+\cdots+a_{k} z^{k}
$$

Recall that the dual of $L_{\omega}(\mathbb{R}) / J_{\omega}(\{0\})$ can be identified with $J_{\omega}(\{0\})^{\perp}$ by using the isomorphism

$$
\theta: J_{\omega}(\{0\})^{\perp} \rightarrow\left(L_{\omega}(\mathbb{R}) / J_{\omega}(\{0\})\right)^{\star}
$$

defined by $\theta(g)=\tilde{g}$ where $g=\tilde{g} \circ \pi\left(g \in J_{\omega}(\{0\})^{\perp}\right)$. We obtain, for $g \in$ $J_{\omega}(\{0\})^{\perp}, t \in \mathbb{R}$,

$$
\left\langle p \star \delta_{t}, g\right\rangle=\left\langle\pi\left(\delta_{t}\right), \tilde{g}\right\rangle=\sum_{i=0}^{k}\left\langle a_{i}, \tilde{g}\right\rangle t^{i}
$$

Let $f \in L_{\omega}(\mathbb{R})$ be such that $\hat{f}_{n}^{(i)}(0)=0$ for $i=0, \ldots, k$ and let $g \in J_{\omega}(\{0\})^{\perp}$. We 
have

$$
\begin{aligned}
\langle f, g\rangle & =\langle f \star p, g\rangle \\
& =\int_{-\infty}^{+\infty} \int_{-\infty}^{+\infty} f(s) p(t-s) g(-t) d t d s \\
& =\int_{-\infty}^{+\infty} f(s) \int_{-\infty}^{+\infty} p(t-s) g(-t) d t d s \\
& =\int_{-\infty}^{+\infty} f(s)\left\langle p \star \delta_{s}, g\right\rangle d s=\int_{-\infty}^{+\infty} f(s)\left(\sum_{j=0}^{k}\left\langle a_{j}, \tilde{g}\right\rangle s^{j}\right) d s \\
& =\sum_{j=0}^{k}\left\langle a_{j}, \tilde{g}\right\rangle \int_{-\infty}^{+\infty} f(s) s^{j} d s=\sum_{j=0}^{k}\left\langle a_{j}, \tilde{g}\right\rangle(-i)^{j} \hat{f}^{(j)}(0)=0 .
\end{aligned}
$$

So $f \in J_{\omega}(\{0\})$.

REMARK. For $k=0$, Theorem 3.2 shows that points satisfy $\omega$-synthesis for all weights $\omega$ such that

$$
\begin{cases}\omega(t)=o(t) & (t \rightarrow+\infty), \\ \log \omega(-t)=o\left(t^{\frac{1}{2}}\right) & (t \rightarrow+\infty),\end{cases}
$$

and this improves [8, Theorem 8.1].

Suppose that $\omega$ satisfies $\lim \inf _{|t| \rightarrow+\infty} \omega(t) /|t|>0$. Set $I=\left\{f \in L_{\omega}(\mathbb{R}): \hat{f}(0)=\right.$ $\left.\hat{f}^{\prime}(0)=0\right\} ; I$ is a closed ideal of $L_{\omega}(\mathbb{R})$ different from $I_{\omega}(\{0\})$ and with $h(I)=\{0\}$. So $\{0\}$ does not satisfy the $\omega$-synthesis.

It follows from this observation that (2) gives, essentially, the best growth conditions on $\omega$ for which points satisfy $\omega$-synthesis.

\section{4. $C_{0}$-groups satisfying some growth conditions}

Let $T$ be a $C_{0}$-group with a generator $A$ and let $\omega$ be a regular weight such that $\|T(t)\|=O(\omega(t))(|t| \rightarrow+\infty)$. For example, $\omega$ can be the weight $\omega_{T}=$ $(\|T(t)\|)_{t \in \mathbb{R}}$.

For $\mu \in M_{\omega}(\mathbb{R})$, we define

$$
\mu(T)=\int_{-\infty}^{+\infty} T(t) d \mu(t)
$$


(Here the integral is the Bochner integral with respect to the strong operator topology [9, Theorem 3.8.2].) The map $\psi: M_{\omega}(\mathbb{R}) \rightarrow \mathscr{L}(X), \mu \rightarrow \mu(T)$ is a continuous algebra homomorphism satisfying

$$
\|\mu(T)\| \leq \sup _{t \in \mathbb{R}} \frac{\|T(t)\|}{\omega(t)}\|\mu\|_{\omega}
$$

Denote by $\sigma(A)$ the spectrum of $A$ [9, Definition 2.16.1]. Let $\mathscr{R}(\lambda)=(\lambda-A)^{-1}$ be the resolvent of $A$, so that $\mathscr{R}$ is defined and analytic on $\mathbb{C} \backslash \sigma(A)$.

Since $\omega$ is a weight, the limits $\lim _{t \rightarrow \pm \infty} \log \omega(t) / t$ exist; and since $\omega$ is regular, $\lim _{|t| \rightarrow+\infty} \log \omega(t) /|t|=0$. In particular, $\|T(n)\|^{1 / n} \rightarrow 1$ as $|n| \rightarrow+\infty$, and so $\sigma(T(1)) \subset \Gamma$, where $\Gamma$ denotes the unit circle. By [9, p. 457] we have $e^{\sigma(A)} \subset$ $\sigma(T(1))$. Hence $\operatorname{Re} \lambda=0$ for all $\lambda \in \sigma(A)$, and we have $[9$, p. 344]

$$
\mathscr{R}(z)= \begin{cases}\int_{0}^{+\infty} T(t) e^{-z t} d t & (\operatorname{Re} z>0), \\ -\int_{-\infty}^{0} T(t) e^{-z t} d t & (\operatorname{Re} z<0) .\end{cases}
$$

LEMMA 4.1. Let $\mu \in M_{\omega}(\mathbb{R})$. Then

$$
\mu(T) x=\lim _{\epsilon \rightarrow 0+} \int_{-\infty}^{+\infty} \hat{\mu}(t)[\mathscr{R}(\epsilon-i t) x-\mathscr{R}(-\epsilon-i t) x] d t,
$$

for all $x$ in the domain of $A^{2}$.

PROOF. Let $x \in X$. For $\epsilon>0$ and $t \in \mathbb{R}$, we set $S_{\epsilon}(t)=e^{-\epsilon|t|} T(-t)$ and

$$
g_{\epsilon}(t)=\int_{-\infty}^{+\infty} S_{\epsilon}(t-s) x d \mu(s)
$$

so that $g_{\epsilon}: \mathbb{R} \rightarrow X$ is continuous. Since $\|T(t)\|=O(\omega(t))(|t| \rightarrow+\infty)$, we have $\int_{-\infty}^{+\infty}\left\|S_{\epsilon}(t)\right\| d t<+\infty$. Hence by Fubini's theorem

$$
\begin{aligned}
\int_{-\infty}^{+\infty}\left\|g_{\epsilon}(t)\right\| d t & \leq \int_{-\infty}^{+\infty} \int_{-\infty}^{+\infty}\left\|S_{\epsilon}(t-s)\right\|\|x\| d|\mu|(s) d t \\
& \leq\|x\|\|\mu\|_{\omega} \int_{-\infty}^{+\infty}\left\|S_{\epsilon}(t)\right\| d t<+\infty .
\end{aligned}
$$

The Fourier transform of $g_{\epsilon}$ is given by the formula

$$
\begin{aligned}
\hat{g}_{\epsilon}(u) & =\int_{-\infty}^{+\infty} e^{-i u t} g_{\epsilon}(t) d t \\
& =\int_{-\infty}^{+\infty} e^{-i u t}\left[\int_{-\infty}^{+\infty} S_{\epsilon}(t-s) x d \mu(s)\right] d t \\
& =\int_{-\infty}^{+\infty}\left[\int_{-\infty}^{+\infty} e^{-i u t} e^{-\epsilon|t-s|} T(s-t) x d t\right] d \mu(s) .
\end{aligned}
$$


We have

$$
\begin{aligned}
\int_{-\infty}^{+\infty} e^{-i u t} e^{-\epsilon|s-t|} T(s-t) x d t & =\int_{-\infty}^{+\infty} e^{i u(v-s)} e^{-\epsilon|v|} T(v) x d v \\
& =e^{-i u s}\left[\int_{-\infty}^{0} e^{(i u+\epsilon) v} T(v) x d v+\int_{0}^{+\infty} e^{(i u-\epsilon) v} T(v) x d v\right] \\
& =e^{-i u s}[\mathscr{R}(\epsilon-i u) x-\mathscr{R}(-\epsilon-i u) x]
\end{aligned}
$$

So

$$
\begin{aligned}
\hat{g}_{\epsilon}(u) & =\hat{\mu}(u)(\mathscr{R}(\epsilon-i u) x-\mathscr{R}(-\epsilon-i u) x) \\
& =-2 \epsilon \hat{\mu}(u) \mathscr{R}(\epsilon-i u) \mathscr{R}(-\epsilon-i u) x .
\end{aligned}
$$

Suppose that $x$ is in the domain of $A$. We have for all $\lambda \notin \sigma(A), \mathscr{R}(\lambda)(\lambda-A) x=x$. So $\mathscr{R}(\lambda) x=(x+\mathscr{R}(\lambda) A x) / \lambda$. It follows from the fact that $\lim _{|t| \rightarrow+\infty} \log \omega(t) /|t|=0$ and [11, Remark 5.4], that there exists a constant $M$ such that $\|\mathscr{R}(\lambda)\| \leq M$ for all $\lambda,|\operatorname{Re} \lambda| \geq \epsilon$. Then we obtain

$$
\|\mathscr{R}(\lambda) x\| \leq(\|x\|+M\|A x\|) /|\lambda|, \quad|\operatorname{Re} \lambda| \geq \epsilon .
$$

Suppose now that $x$ is in the domain of $A^{2}$. We deduce from the above inequality that

$$
\begin{aligned}
\|\mathscr{R}(\epsilon-i u) \mathscr{R}(-\epsilon-i u) x\| & \leq \frac{1}{\left(\epsilon^{2}+u^{2}\right)^{1 / 2}}(\|\mathscr{R}(-\epsilon-i u) x\|+M\|\mathscr{R}(-\epsilon-i u) A x\|) \\
& \leq \frac{1}{\left(\epsilon^{2}+u^{2}\right)}\left(\|x\|+2 M\|A x\|+M^{2}\left\|A^{2} x\right\|\right) .
\end{aligned}
$$

This estimate and the fact that $\hat{\mu}$ is a bounded function show that $\hat{g}_{\epsilon}$ is integrable. Since $g_{\epsilon}$ is continuous, we obtain by the inverse Fourier transform, for all $t \in \mathbb{R}$,

$$
\begin{aligned}
g_{\epsilon}(t) & =\frac{1}{2 \pi} \int_{-\infty}^{+\infty} e^{i t u} \hat{g}_{\epsilon}(u) d u \\
& =\frac{1}{2 \pi} \int_{-\infty}^{+\infty} \hat{\mu}(s) e^{i s t}(\mathscr{R}(\epsilon-i s) x-\mathscr{R}(-\epsilon-i s) x) d s .
\end{aligned}
$$

We have

$$
g_{\epsilon}(0)=\int_{-\infty}^{+\infty} e^{-\epsilon|s|} T(s) x d \mu(s),
$$

which implies that $\mu(T) x=\lim _{\epsilon \rightarrow 0} g_{\epsilon}(0)$, and this proves the lemma.

The following result is certainly known but we have not been able to find a precise reference. 
THEOREM 4.2. Let $T$ be a $C_{0}$-group with a generator $A$ and let $\omega$ be a regular weight such that $\|T(t)\|=O(\omega(t))(|t| \rightarrow+\infty)$. Let

$$
\varphi: L_{\omega}(\mathbb{R}) \rightarrow L(X), \quad f \rightarrow f(T)=\int_{-\infty}^{+\infty} f(t) T(t) d t .
$$

Then $\operatorname{ker} \varphi$ is a closed ideal of $L_{\omega}(\mathbb{R})$ and $h(\operatorname{ker} \varphi)=i \sigma(A)$.

PROOF. Note that $\varphi$ is the restriction of $\psi$ to $L_{\omega}(\mathbb{R})$ and so $\varphi$ is a continuous algebra homomorphism. So $\operatorname{ker} \varphi$ is a closed ideal of $L_{\omega}(\mathbb{R})$.

Set $\mathscr{A}=L_{\omega}(\mathbb{R})+\mathbb{C} \delta_{0} ; \operatorname{ker} \varphi$ is also a closed ideal of $\mathscr{A}$. The set of characters of $\mathscr{A}$ can be identified with the set $\left\{\chi_{x}: x \in \mathbb{R}\right\} \cup\left\{\chi_{\infty}\right\}$ where $\chi_{x}(\mu)=\hat{\mu}(x)$ and $\chi_{\infty}(\mu)=\lim _{|x| \rightarrow \infty} \hat{\mu}(x)(\mu \in \mathscr{A})$. So, the set of characters of $\mathscr{A} / \operatorname{ker} \varphi$ can be indentified with the set $\left\{\chi_{x}: x \in h(\operatorname{ker} \varphi)\right\} \cup\left\{\chi_{\infty}\right\}$. Since $\operatorname{ker} \varphi \subset \operatorname{ker} \psi \cap \mathscr{A}$ there exists a homomorphism $\tilde{\psi}: \mathscr{A} / \operatorname{ker} \varphi \rightarrow L(X)$ such that $\tilde{\psi} \circ \pi=\psi$, where $\pi$ is the canonical surjection from $\mathscr{A}$ onto $\mathscr{A} / \operatorname{ker} \varphi$.

Let $v(x)=e^{-x}(x \geq 0)$ and $v(x)=0(x<0)$, so that $v \in L_{\omega}(\mathbb{R})$. The spectrum $\sigma(\pi(v))$ of $\pi(v)$ in $\mathscr{A} / \operatorname{ker} \varphi$ is given by

$$
\sigma(\pi(v))=\left\{(1+i x)^{-1}: x \in h(\operatorname{ker} \varphi)\right\} \cup\{0\} .
$$

We have

$$
v(T)=\int_{0}^{+\infty} T(t) e^{-t} d t
$$

and the relation between the Carlemann transform of $T$ and the resolvent of $A$, recalled in the beginning of this paragraph, implies that $v(T)=(I-A)^{-1}$. Since $v(T)=\varphi(v)=\tilde{\psi}(\pi(v))$, we have $\sigma(v(T)) \subset \sigma(\pi(v))$. So if $x \in \sigma(A)$ then $x \neq 1,1 /(1-x) \in \sigma(v(T)) \subset \sigma(\pi(v))$, and $1 /(1-x)=1 /(1+i y)$ with $y \in h(\operatorname{ker} \varphi)$, and so $i x=y \in h(\operatorname{ker} \varphi)$. Thus $i \sigma(A) \subset h(\operatorname{ker} \varphi)$.

For $n \geq 1$, set $e_{n}=n 1_{[0,1 / n]}$, where $1_{[0,1 / n]}$ is the characteristic function of the interval $[0,1 / n]$. We have for all $f \in L_{\omega}(\mathbb{R}),\left\|f \star e_{n}-f\right\|_{\omega} \rightarrow 0$ as $n \rightarrow+\infty[13$, Proposition 1.1].

Let $I$ be the closure of the set of all functions $f \in L_{\omega}(\mathbb{R})$ such that $\hat{f}$ has compact support; $I$ is a closed ideal of $L_{\omega}(\mathbb{R})$ such that $h(I)=\phi$. Wiener's Tauberian theorem for regular Beurling algebras shows that $I=L_{\omega}(\mathbb{R})$ (see [2] and [5]). It follows that for $n \geq 1$, there exists $k_{n} \in L_{\omega}(\mathbb{R})$ such that the support of $\hat{k}_{n}$ is compact and $\left\|k_{n}-e_{n}\right\|_{\omega}<1 / n$. Thus $\left\|f \star k_{n}-f\right\|_{\omega} \rightarrow 0$ as $n \rightarrow+\infty\left(f \in L_{\omega}(\mathbb{R})\right)$.

Fix now $f \in L_{\omega}(\mathbb{R})$ and let $x$ be in the domain of $A^{2}$, which we denote by $\mathscr{D}\left(A^{2}\right)$. It follows from Lemma 4.1 that

$$
\left(f \star k_{n}\right)(T) x=\frac{1}{2 \pi} \lim _{\epsilon \rightarrow 0^{+}} \int_{-\infty}^{+\infty} \hat{f}(t) \hat{k}_{n}(t)[\mathscr{R}(\epsilon-i t) x-\mathscr{R}(-\epsilon-i t) x] d t .
$$


Suppose that $f$ vanishes on a neighbourhood of $i \sigma(A)$. Then it is clear that $\left(f \star k_{n}\right)(T) x=0$. Since $\mathscr{D}\left(A^{2}\right)$ is dense in $X$ [11, Theorem 2.7] and since $\left(f \star k_{n}\right)(T)$ is a bounded operator, we have $\left(f \star k_{n}\right)(T)=0$. Using the inequality

$$
\left\|f(T)-\left(f \star k_{n}\right)(T)\right\| \leq \sup _{t \in \mathbb{R}} \frac{\|T(t)\|}{\omega(t)}\left\|f \star k_{n}-f\right\|_{\omega}
$$

we see that $f(T)=0$.

The map $\varphi$ is continuous and so $J_{\omega}(i \sigma(A)) \subseteq \operatorname{ker} \varphi$. Thus $h(\operatorname{ker} \varphi) \subseteq h\left(J_{\omega}(i \sigma(A))\right.$. Since the algebra $L_{\omega}(\mathbb{R})$ is regular, $h\left(J_{\omega}(i \sigma(A))\right)=i \sigma(A)$, which concludes the proof of the theorem.

THEOREM 4.3. Let $k \geq 0$ be an integer and let $T$ be a $C_{0}$-group with a generator A. Assume that the following conditions hold:

(i) $\|T(t)\|=o\left(t^{k+1}\right) \quad(t \rightarrow+\infty)$;

(ii) $\log ^{+}\|T(-t)\|=o\left(t^{1 / 2}\right) \quad(t \rightarrow+\infty)$.

If $\sigma(A)=\{\lambda\}$, then $A$ is bounded and $(A-\lambda)^{k+1}=0$.

PROOF. We have $\operatorname{Re} \lambda=0$ and multiplying $T(t)$ by $e^{-\lambda t}$ we can assume without loss of generality that $\lambda=0$.

Set $\omega(t)=\max \left(\|T(t)\|,(1+|t|)^{k}\right),(t \in \mathbb{R}) ; \omega$ is a weight satisfying the three conditions of Theorem 3.2 and we have $\|T(t)\|=O(\omega(t))(t \rightarrow+\infty)$.

Let

$$
u(t)= \begin{cases}0 & \text { if }|t| \geq 1, \\ c e^{-1 /\left(1-|t|^{2}\right)} & \text { if }|t|<1 .\end{cases}
$$

the constant $c$ is chosen such that $\int_{-1}^{1} u(t) d t=1$. The function $u$ is infinitely differentiable and $\operatorname{Supp}(u)=[-1,1]$. Set, for $n \geq 1$, and for $t \in \mathbb{R}, u_{n}(t)=n u(n t)$ and $e_{n}(t)=u_{n}^{(k+1)}(t)$. Clearly, $\operatorname{Supp}\left(e_{n}\right)=[-1 / n, 1 / n]$ and $e_{n} \in L_{\omega}(\mathbb{R})$. We have $\hat{e}_{n}(t)=(i t)^{k+1} \hat{u}_{n}(t)$. Thus $\hat{e}_{n}^{(i)}(0)=0$ for $i=0,1, \ldots, k$ and Theorem 3.2 shows that $e_{n}$ satisfies $\omega$-synthesis for $\{0\}$. It follows from Theorem 4.2 that $h(\operatorname{ker} \varphi)=\{0\}$, so that $e_{n} \in \operatorname{ker} \varphi$. So $e_{n}(T)=0$.

Let $x$ be in the domain of $A^{k+1}$. The map $h_{x}: \mathbb{R} \rightarrow X, t \mapsto T(t) x$ is $(k+1)$-times continuously differentiable and $h_{x}^{(k+1)}(t)=T(t) A^{k+1} x$ [9, Theorem 11.5.3].

We have

$$
e_{n}(T) x=\int_{-\infty}^{+\infty} e_{n}(t) h_{x}(t) d t
$$

applying integration by parts $(k+1)$-times we obtain

$$
e_{n}(T)=(-1)^{k+1} \int_{-\infty}^{+\infty} u_{n}(t) h_{x}^{(k+1)}(t) d t
$$


So

$$
\begin{aligned}
\left\|e_{n}(T) x-(-1)^{k+1} A^{k+1} x\right\| & =\left\|\int_{-\infty}^{+\infty} u_{n}(t)\left(T(t) A^{k+1} x-A^{k+1} x\right) d t\right\| \\
& =\left\|\int_{-\infty}^{+\infty} \frac{1}{n} u_{n}\left(\frac{t}{n}\right)\left(T\left(\frac{t}{n}\right) A^{k+1} x-A^{k+1} x\right) d t\right\| \\
& =\left\|\int_{-1}^{1} u(t)\left(T\left(\frac{t}{n}\right) A^{k+1} x-A^{k+1} x\right) d t\right\| \\
& \left.\leq \sup _{-1 \leq t \leq 1} \| T\left(\frac{t}{n}\right) A^{k+1} x-A^{k+1} x\right) \| .
\end{aligned}
$$

Since the map $t \mapsto T(t) A^{k+1} x$ is continuous, the latter quantity converges to 0 as $n \rightarrow+\infty$. Thus $e_{n}(T) x \rightarrow(-1)^{k+1} A^{k+1} x$ as $n \rightarrow+\infty$. Since $e_{n}(T)=0$, we obtain $A^{k+1} x=0$. Hence $A^{k+1}=0$. It follows from $[9$, p. 56] that $A$ is bounded.

We will make precise the relation between Theorem 3.2 and Theorem 4.3 in the case $k=0$. We will say that a regular weight $\omega$ satisfies property $(P)$ if :

(P) For all $C_{0}$-groups $T$ with $\sigma(A)=\{0\}$ and $\|T(t)\|=O(\omega(t))(|t| \rightarrow+\infty)$, we have $A=0$.

THEOREM 4.4. Let $\omega$ be a regular weight. Then $\omega$ satisfies property $(P)$ if and only if points satisfy $\omega$-synthesis.

PROOF. We will use the notations introduced in the proof of Theorem 3.2. Suppose that $\omega$ satisfies property (P). The $C_{0}$-group $\left(\pi\left(\delta_{t}\right)\right)_{t \in \mathbb{R}} \subseteq L_{\omega}(\mathbb{R}) / J_{\omega}(\{0\})$ is such that $\sigma(u)=0$ and $\left\|\pi\left(\delta_{t}\right)\right\| \leq\|p\|_{\omega} \omega(t)(t \in \mathbb{R})$. Hence $u=0$ and $\pi\left(\delta_{t}\right)=\pi\left(\delta_{0}\right)(t \in \mathbb{R})$.

Let $f \in I_{\omega}(\{0\})$ and let $g \in J_{\omega}(\{0\})^{\perp}$. Then

$$
\begin{aligned}
\langle f, g\rangle & =\langle f \star p, g\rangle=\int_{-\infty}^{+\infty} f(s)\left\langle p \star \delta_{s}, g\right\rangle d s \\
& =\int_{-\infty}^{+\infty} f(s)\left\langle\pi\left(\delta_{s}\right), \tilde{g}\right\rangle d s=\langle\pi(p), \tilde{g}) \hat{f}(0)=0 .
\end{aligned}
$$

Thus $g \in I_{\omega}(\{0\})^{\perp}$. It follows that $I_{\omega}(\{0\})=J_{\omega}(\{0\})$.

Conversely, suppose that $\{0\}$ satisfies $\omega$-synthesis and let $T$ be such that $\sigma(A)=\{0\}$ and $\|T(t)\|=O(\omega(t))(|t| \rightarrow+\infty)$.

Consider the function $\varphi: L_{\omega}(\mathbb{R}) \rightarrow \mathscr{L}(X), f \mapsto f(T)$. By Theorem 4.2, $h(\operatorname{ker} \varphi)=\{0\}$. Since $\{0\}$ satisfies $\omega$-synthesis, $\operatorname{ker} \varphi=I_{\omega}(\{0\})$. For each integer $n \geq 1$ let $e_{n}$ be the function defined in the proof of Theorem 4.3 for $k=0$. We have $e_{n}(T) x \rightarrow-A x$ for all $x$ in the domain of $A$. Since $\hat{e}_{n}(0)=0, e_{n}(T)=0$. Hence $A=0$. 


\section{The discrete case}

We now give a result, analogous to Theorem 4.3 as announced in the introduction.

THEOREM 5.1. Let $k \geq 0$ be an integer and let $R$ be a bounded operator on a Banach space with spectrum equal to $\{\lambda\}$ and such that

(i) $\left\|R^{n}\right\|=o\left(n^{k+1}\right)(n \rightarrow+\infty)$;

(ii) $\log ^{+}\left\|R^{-n}\right\|=o\left(n^{1 / 2}\right)(n \rightarrow+\infty)$.

Then $(R-\lambda)^{k+1}=0$.

PROOF. We have $|\lambda|=1$ and we can assume without loss of generality that $\lambda=1$. Let

$$
A=\log R=\sum_{n \geq 1} \frac{(-1)^{n-1}}{n}(R-I)^{n} .
$$

(This series is convergent since $\lim _{n \rightarrow+\infty}\left\|(R-I)^{n}\right\|^{1 / n}=0$.) Set $T(t)=e^{t A}(t \in \mathbb{R})$. It is well-known and easy to check that $\sigma(A)=\{0\}$ and $T(n)=R^{n}(n \in \mathbb{Z})$.

For all $t \in \mathbb{R}$ there exists $n \in \mathbb{Z}$ such that $|t-n|<1$ and $|n| \leq|t|$. We have

$$
\|T(t)\| \leq\|T(t-n)\|\|T(n)\| \leq \sup _{-1 \leq s \leq 1}\|T(s)\|\|T(n)\| \leq e^{\|A\|}\|T(n)\| .
$$

Since $|n| \leq|t|$, we see that the group $T$ satisfies conditions (i) and (ii) of Theorem 4.3. Hence $A^{k+1}=0$. We have

$$
A^{k+1}=(R-I)^{k+1}\left(\sum_{n \geq 1} \frac{(-1)^{n-1}}{n}(R-I)^{n-1}\right)^{k+1} .
$$

Since the operator $\sum_{n \geq 1}\left((-1)^{n-1} / n\right)(R-I)^{n-1}$ is invertible, we obtain $(R-I)^{k+1}=0$.

REMARK. Let $\omega$ be a weight on $\mathbb{Z}$ and let

$$
A_{\omega}(\Gamma)=\left\{f \in \mathscr{C}(\Gamma): \sum_{n \in \mathbb{Z}}|\hat{f}(n)| \omega(n)<+\infty\right\}
$$

where $\Gamma$ is the unit circle. As in Definition 3.1 we can define the $\omega$-spectral synthesis property for a closed subset of $\Gamma$ [14]. By the same methods we can prove results analogous to Theorems 3.2 and 4.4 in the discrete case. The result analogous to Theorem 4.2 is given in [14].

Finally we give examples to show that conditions (i) and (ii) in Theorem 4.3 and Theorem 5.1 are essentially the best possible. 
EXAMPLE 1. Let $k \geq 0$ be an integer and let $A: \mathbb{C}^{k+2} \rightarrow \mathbb{C}^{k+2}$ be the operator defined by the matrix

$$
\left(\begin{array}{ccccc}
0 & 1 & & & \\
& \cdot & \cdot & 0 & \\
& & \cdot & \cdot & \\
& 0 & & \cdot & 1 \\
& & & & 0
\end{array}\right)
$$

with respect to the canonical basis of $\mathbb{C}^{k+2}$. Define $T(t)=e^{t A}(t \in \mathbb{R})$. A direct calculation gives $\|T(t)\|=O\left(|t|^{k+1}\right)(|t| \rightarrow+\infty)$. But is is clear that $A^{k+1} \neq 0$.

EXAMPLE 2. Let $H^{2}$ be the Hardy space, let $\theta(z)=e^{(z+1) /(z-1)},(|z|<1)$ and let $\mathscr{H}=H^{2} \Theta \theta H^{2}$. Denote by $P_{\mathscr{H}}$ the orthogonal projection on $\mathscr{H}$ and set $R(f)=P_{\mathscr{H}}(z f)(f \in \mathscr{H})$. Then $R$ is a contraction with spectrum $\{1\}$ such that $\log \left\|R^{-n}\right\|=O\left(n^{1 / 2}\right)(n \rightarrow+\infty)\left[11\right.$, Remark 2.c]. But $(R-I)^{k} \neq 0$ for all integers $k \geq 0$.

If we set

$$
A=\sum_{n \geq 1} \frac{(-1)^{n}}{n}(R-I)^{n},
$$

then $\sigma(A)=\{0\}$ and $A$ is the infinitesimal generator of a norm-continuous group $T$ which satisfies $\|T(t)\|=O(1)(t \rightarrow+\infty), \log ^{+}\|T(-t)\|=O\left(t^{1 / 2}\right)(t \rightarrow+\infty)$ and $A^{k} \neq 0$ for all integers $k \geq 0$ (see the proof of Theorem 5.1).

\section{Acknowledgements}

I wish to thank Professors W. Arendt and J. Esterle for helpful conversations concerning this work.

\section{References}

[1] G. R. Allan and T. J. Ransford, 'Power-dominated elements in a Banach algebra', Studia Math. 94 (1989), 63-79.

[2] A. Atzmon, 'Operators which are annihilated by analytic functions and invariant subspaces', Acta Math. 144 (1980), 27-63.

[3] A. Beurling, 'Sur les integrales de Fourier absolument convergentes et leur application à une transformation fonctionnelle', in: Neuvième Cong. Math. Scandinaves, Helsinki 1938 (Tryekeri, Helsinki, 1939) pp. 199-210.

[4] R. P. Boas, Entire functions (Academic Press, New York, 1954).

[5] H. G. Dales and W. K. Hayman, 'Esterle's proof of the Tauberian theorem for Beurling algebras', Ann. Inst. Fourier (Grenoble) 31 (1981), 141-150. 
[6] J. Esterle, 'Distributions on Kronecker sets, strong form of uniqueness and closed ideals of $A^{+}$, J. Reine Angew. Math. 450 (1994), 43-82.

[7] J. Esterle, E. Strouse and F. Zouakia, 'Stabilité asymptotique de certains semi-groupes d'operateurs', J. Operator Theory 28 (1992), 203-227.

[8] V. P. Gurarii, 'Harmonic analysis in spaces with weights', Trans. Moscow Math. Soc. 35 (1979), 21-75.

[9] E. Hille and R. S. Phillips, Functional analysis and semi-groups, Amer. Math. Soc. Colloq. Publ. 31 (Amer. Math. Soc., Providence, 1957).

[10] Y. Katznelson, An introduction to harmonic analysis (Wiley, New York, 1968).

[11] A. Pazy, Semigroups of linear operators and applications to partial differential equations (Springer, New York, 1983).

[12] H. H. Schaefer, M. Wolf and W. Arendt, 'On lattice isomorphisms with positive real spectrum and group operators', Math. Z. 164 (1978), 115-123.

[13] M. Zarrabi, 'Ensembles de synthèse pour certaines algèbres de Beurling', Rev. Roumaine Math. Pures Appl. 35 (1990), 385-396.

[14] —_, 'Contractions à spectre dénombrable et propriétés d'unicité des fermés dénombrables du cercle', Ann. Inst. Fourier (Grenoble) 43 (1993), 251-263.

\section{U.F.R de Mathématiques et Informatique}

Université Bordeaux I

351 , cours de la Liberation

33405 Talence

France

e-mail: zarrabi@math.u-bordeaux.fr 